\title{
Nitratos y nitritos, la doble cara de la moneda
}

\author{
Nitrates and nitrites, the two-sided coin \\ Nitratos e nitritos, o lado duplo da moeda
}

Mateo Londoño Pereira ${ }^{1 *}$, Briana Davahiva Gómez Ramírez ${ }^{1,2}$

Recibido: 28 de agosto de 2020. Aceptado para publicación: 10 de octubre de 2020

Publicado en línea: 29 de octubre de 2020

https://doi.org/10.35454/rncm.v4n1.202

\begin{abstract}
Resumen
Los nitratos y nitritos son compuestos químicos inorgánicos derivados del nitrógeno, que se encuentran naturalmente en alimentos vegetales y de manera adicionada en algunos productos cárnicos procesados. Su presencia en la dieta se ha relacionado históricamente con un alto riesgo de desarrollar cáncer en diferentes tejidos debido a su capacidad de transformarse en $\mathrm{N}$-nitrosaminas, altamente carcinogénicas. El objetivo de este artículo es revisar el impacto que tiene para la salud humana la ingesta de nitratos y nitritos alimentarios, en relación con su procedencia de fuentes naturales o de productos procesados a los que han sido añadidos como aditivos químicos.

A pesar de las altas concentraciones de nitratos en alimentos vegetales, no hay justificación válida para restringir su ingesta por un posible incremento en el riesgo de padecer cáncer. Esto se debe a la facultad de los compuestos antioxidantes propios de los alimentos de origen vegetal para inducir la formación de óxido nítrico e inhibir las reacciones de nitrosación y formación de nitrosaminas, lo que tiene efectos favorables para la salud cardiovascular, cognitiva y en el rendimiento deportivo. Se destaca, además, que las dietas con mayor evidencia en la prevención de enfermedades crónicas como el cáncer son particularmente altas en nitratos naturales. Se recomienda precaución en el consumo de nitritos y nitratos provenientes de alimentos procesados, por un posible mayor riesgo de cáncer.
\end{abstract}

Palabras clave: nitratos, nitritos, óxido nítrico, nitrosaminas, alimentos, evaluación en salud.

\section{Abstract}

Nitrates and nitrites are inorganic chemical compounds derived from nitrogen found naturally in vegetable foods and as additives in some processed meat products. Their presence in the diet has historically been linked to a high risk of developing cancer in different sites due to their ability to transform into highly carcinogenic $\mathrm{N}$-nitrosamines. The objective of this review article is to review the impact that the intake of dietary nitrates and nitrites has on human health in relation to their origin, either from natural sources or processed products to which they have been added as chemical additives.

Despite the high concentrations of nitrates in vegetables, there is no valid justification for restricting their intake due to a possible increased the risk of cancer. This is due to the ability of the antioxidant compounds found in foods of plant origin to induce the formation of nitric oxide and inhibit nitrosation reactions and formation of nitrosamine, thus inducing favorable effects on cardiovascular and cognitive health and on sports performance. We also highlight that the diets with the greatest evidence regarding the prevention of chronic diseases, such as cancer, are those particularly high in natural nitrates. Caution is recommended in the intake of nitrites and nitrates from processed foods, due to a potentially increased risk of cancer.

Keywords: Nitrates; Nitrites; Nitric oxide; Nitrosamines; Food; Health evaluation.

\section{Resumo}

Os nitratos e nitritos são compostos químicos inorgânicos derivados do nitrogênio, que são encontrados naturalmente em alimentos vegetais e em adição em alguns produtos cárneos processados. Sua presença na dieta tem sido historicamente relacionada ao alto risco do desenvolvimento de câncer em diferentes tecidos, devido a sua capacidade de se transformar em N-nitrosaminas, altamente carcinogênicas. O objetivo deste artigo é revisar o impacto que tem na saúde humana a ingestão alimentar de nitratos e nitritos, em relação à sua origem a partir de fontes naturais ou produtos processados aos quais foram adicionados como aditivos químicos. Apesar das altas concentrações de nitratos nos alimentos vegetais, não há justificativa válida para restringir sua ingestão devido a um possível aumento do risco de desenvolver o câncer. Isto deve-se à capacidade dos compostos antioxidantes encontrados nos alimentos de origem vegetal para induzir a formação de óxido nítrico e inibir as reações de nitrosação e formação de nitrosaminas, permitindo efeitos favoráveis para a saúde cardiovascular, cognitiva e no desempenho esportivo. Ressalta-se também que as dietas com maior evidência na prevenção de doenças crônicas como o câncer são particularmente ricas em nitratos naturais. Recomenda-se cautela no consumo de nitritos e nitratos de alimentos processados, devido a um possível maior risco de desenvolver câncer.

Palavras-chave: nitratos, nitritos, óxido nítrico, nitrosaminas, alimentos, avaliação em saúde 
1. Escuela de Nutrición y Dietética, Universidad de Antioquia. Medellín, Colombia.

*Correspondencia: Mateo Londoño Pereira

mateo.londonop@udea.edu.co

\section{INTRODUCCIÓN}

La ingesta y exposición a iones de nitrato $\left(\mathrm{NO}_{3}^{-}\right)$y nitrito $\left(\mathrm{NO}_{2}^{-}\right)$en la dieta ha sido amplia e históricamente controversial. Por una parte, es bien reconocido que los nitritos adicionados en procesados cárnicos pueden transformarse en nitrosaminas carcinogénicas, por lo que se ha sugerido precaución en su consumo ${ }^{(1)}$. Por otra parte, se ha manifestado que los procesados cárnicos no son las únicas fuentes alimentarias que pueden aportar estos compuestos nitrosos. De hecho, los alimentos de origen vegetal como las verduras, frutas y legumbres, naturalmente tienen compuestos nitrogenados como los nitratos, que a nivel orgánico pueden seguir diferentes rutas metabólicas, algunas de ellas con impacto favorable para la salud ${ }^{(2)}$.

Esta disyuntiva lleva a que los profesionales del área de la salud no tengan claridad sobre las diferencias entre estos compuestos nitrosos de origen vegetal y los adicionados de origen animal, lo que ha llevado, incluso, a que se desestime el consumo de vegetales con alto contenido de nitratos, porque se piensa que, sin importar la fuente, ambos derivarán en nitrosaminas y, por tanto, su impacto en la salud será negativo. Por consiguiente, el objetivo de esta revisión es determinar el impacto que tiene para la salud humana la ingesta de nitratos y nitritos alimentarios, en relación con la procedencia de fuentes naturales o de productos procesados a los que han sido añadidos como aditivos químicos.

Para dar cumplimiento a este objetivo se revisaron las bases de datos Medline y Science Direct, utilizando los siguientes términos de búsqueda: "Nitrate, nitrite" AND "Human Health", "Nitrate, nitrite" AND "Nitric Oxide", "Nitrate, nitrite" AND "N-nitroso Compounds", "Nitrate,nitrite" AND "Cardiovascular Health", "Nitric Oxide Related Health Effects", "Vegetable Nitrates Content", "Dietary Nitrates, nitrites", "Nitrates, nitrites" AND "Cancer Risk". Se evaluaron los estudios publicados en revistas en inglés, con línea de tiempo no superior a diez años y sin límites en el tipo de estudio (revisión, observacional, experimental). Finalmente, se
2. Grupo de Investigación en Alimentación y Nutrición Humana, Universidad de Antioquia. Medellín, Colombia.

seleccionaron aquellos que se consideró que cumplían con el objetivo de la revisión.

\section{ORÍGENES Y EXPOSICIÓN A COMPUESTOS N-NITROSOS}

El nitrógeno molecular (N2) es químicamente inerte y debe fijarse para ser biológicamente activo. Esto sucede a través del ciclo del nitrógeno $\left(\mathrm{N}_{2}\right)$, en el que el $\mathrm{N}_{2}$ se fija a las plantas como amoníaco, $\mathrm{NO}_{2}-\mathrm{y} \mathrm{NO}_{3}$-, apoyado por bacterias nitrificantes $y$, de este modo, puede ser utilizable por la planta ${ }^{(3)}$. Durante la producción agrícola, los suelos se complementan con fertilizantes de amonio (nitrato de amonio, fosfatos de amonio, sulfato de amonio, sales de nitrato, urea y otras formas orgánicas de $\mathrm{N}_{2}$ ), que se convierte rápidamente en nitrato por bacterias nitrificantes. También se usan otros compuestos orgánicos que son una excelente fuente de $\mathrm{N}_{2}$ como el estiércol animal y residuos de leguminosas, que contribuyen al mejoramiento del suelo, y aproximadamente la mitad de este se convertirá en nitrato ${ }^{(3)}$. Sin embargo, la aplicación de fertilizantes en cantidades que van más allá de la capacidad del cultivo para usarlos o de ciertos productos químicos para el control de malezas puede resultar en una acumulación alta de nitrato, particularmente si algún otro nutriente esencial no es adecuado, como el fósforo, el potasio y el calcio ${ }^{(3)}$.

Entonces, más del $90 \%$ del $\mathrm{N}_{2}$ absorbido por las plantas está en forma de nitrato y es necesario para el buen crecimiento de la planta. No obstante, existen amplias variaciones en su contenido entre las especies e incluso variaciones entre las regiones geográficas en las que se cultivan $^{(3)}$. El cientifico Bryan NS documentó que un análisis de varios vegetales de cinco ciudades diferentes de Estados Unidos reveló que el contenido de nitritos y nitratos puede variar hasta 500 veces en el mismo vegetal dependiendo de la ciudad ${ }^{(3,4)}$. Bedale $\mathrm{W}$ reportó una diferencia de 8 veces en los niveles de nitrato de lechuga según la ubicación y las condiciones de crecimiento $^{(5)}$. Las concentraciones de nitrato en alimentos vegetales pueden verse influenciadas por factores como el tipo de 
suelo, momento de aplicación de los fertilizantes nitrogenados, la temperatura y exposición a la luz solar de los cultivos, las relaciones con el agua y las condiciones de conservación y procesamiento de los alimentos ${ }^{(1,4)}$.

Por la anterior razón, la principal fuente de exposición al nitrato por parte de los seres humanos es a través del consumo de verduras, que pueden contribuir al $85 \%$ de la ingesta diaria ${ }^{(3)}$. Algunos vegetales como el rábano, lechuga, remolacha, espinaca y rúcula contienen niveles altos de nitratos ${ }^{(4,6)}$. En la Tabla 1 se observan las estimaciones de la cantidad de nitratos y nitritos de diferentes alimentos por cada 100 gramos de producto, por medio de la adaptación de los datos de Griesenbeck JS y colaboradores ${ }^{(7)}$ y Karwowska My colaboradores $^{(8)}$. En este sentido, los niveles de nitritos en las verduras son más bajos que los niveles de nitrato, y el agua potable generalmente tiene concentraciones insignificantes de nitrito. Algunos tipos de agua potable pueden contener estos compuestos debido a la desinfección del agua con dicloramina, que puede reaccionar para producir nitritos ${ }^{(9)}$. Además, existe la reducción de nitrato a nitrito por las bacterias comensales en la len-

Tabla 1. Estimaciones de nitratos y nitritos en los alimentos

\begin{tabular}{|c|c|c|}
\hline Alimentos & $\begin{array}{l}\text { Nitratos } \\
(\mathrm{mg} / 100 \mathrm{~g})\end{array}$ & $\begin{array}{c}\text { Nitritos } \\
(\mathrm{mg} / 100 \mathrm{~g})\end{array}$ \\
\hline \multicolumn{3}{|l|}{ Productos lácteos } \\
\hline Queso & 1,4 & 5,0 \\
\hline Helado & 0,3 & 15,0 \\
\hline Leche entera & 0,1 & 0,5 \\
\hline Yogur & 0,1 & 23,3 \\
\hline \multicolumn{3}{|l|}{ Frutas y verduras } \\
\hline Melón & 9,5 & 0,4 \\
\hline Manzanas o peras frescas & 1,0 & 0,7 \\
\hline Jugo de naranja & 2,0 & 0,0 \\
\hline Naranjas & 2,0 & 0,0 \\
\hline $\begin{array}{l}\text { Otras frutas frescas, } \\
\text { congeladas o enlatadas }\end{array}$ & 2,6 & 0,8 \\
\hline Tomates & 3,2 & 1,3 \\
\hline Brócoli & 34,1 & 0,9 \\
\hline Repollo, coliflor y coles & 17,2 & 1,1 \\
\hline Zanahorias crudas & 16,7 & 0,8 \\
\hline Aguacate & 2,6 & 0,8 \\
\hline Rábano & 129,8 & SD \\
\hline Apio & 110,3 & SD \\
\hline Lechuga & 132,4 & SD \\
\hline Acelga & 169,0 & SD \\
\hline Remolacha & 144,6 & SD \\
\hline Espinaca cocida & 209,9 & 0,1 \\
\hline
\end{tabular}

\begin{tabular}{|c|c|c|}
\hline Alimentos & $\begin{array}{l}\text { Nitratos } \\
(\mathrm{mg} / 100 \mathrm{~g})\end{array}$ & $\begin{array}{l}\text { Nitritos } \\
(\mathrm{mg} / 100 \mathrm{~g})\end{array}$ \\
\hline \multicolumn{3}{|c|}{ Tubérculos, plátanos, legumbres y oleaginosas } \\
\hline Plátanos & 1,9 & 1,8 \\
\hline Ñame u otros tubérculos & 4,6 & 1,3 \\
\hline $\begin{array}{l}\text { Papas al horno, hervidas o } \\
\text { en puré }\end{array}$ & 14,5 & 0,7 \\
\hline Frijoles o lentejas crudos & 0,8 & 30,9 \\
\hline Arvejas verdes & 17,2 & 1,0 \\
\hline Nueces & 0,6 & 0,0 \\
\hline \multicolumn{3}{|c|}{ Productos de cereales y de legumbres } \\
\hline $\begin{array}{l}\text { Galletas dulces, tortas, } \\
\text { croissant y muffin }\end{array}$ & 0,4 & 10,0 \\
\hline Arroz o pasta & 1,6 & 13,0 \\
\hline Tortilla & 1,6 & 10,0 \\
\hline Pan blanco y galletas saladas & 1,6 & 10,0 \\
\hline Bebida de soya & 5,5 & 1,7 \\
\hline \multicolumn{3}{|l|}{ Carnes y productos cárnicos } \\
\hline Carne de res o cerdo & 5,8 & 22,2 \\
\hline Tocino & 8,8 & 32,9 \\
\hline Pollo o pavo & 0,6 & 80,0 \\
\hline Huevos & 0,5 & 30,0 \\
\hline Pescado & 0,9 & 33,3 \\
\hline $\begin{array}{l}\text { Carnes procesadas: salchicha, } \\
\text { salami, jamón }\end{array}$ & 6,1 & 18,2 \\
\hline
\end{tabular}

SD: sin dato. Modificado de ${ }^{(7,8)}$. 
gua; por lo anterior, la cantidad de nitrito que se ingiere en la saliva es del orden de $13-180 \mathrm{mg} / \mathrm{día}^{(5)}$.

También existe una variedad de compuestos $\mathrm{N}$-nitrosos que tienen otros orígenes, dentro de las que se encuentran las nitrosaminas y sus precursores, que se pueden encontrar en diversos productos de consumo alimentario (procesados cárnicos y de pescado, cerveza), cosméticos y pesticidas. Además, se encuentran bajo la presencia de humo de cigarrillo o endógenamente en la boca o el estómago si el alimento ingerido tiene precursores de nitrosamina, debido a que los nitratos o nitritos se combinan con aminas ${ }^{(10)}$. Los compuestos $\mathrm{N}$-nitrosos identificados con mayor frecuencia en alimentos son $\mathrm{N}$-nitrosodimetilamina (NDMA), $\mathrm{N}$-nitrosopirrolidina (NPYR), N-nitrosopiperidina (NPIP) y N-nitrosotiazolidina $(\mathrm{NTHZ})^{(10)}$. Estos pueden formarse en la elaboración de procesados cárnicos como consecuencia de la adición de sales de nitrato/ nitrito, interconvertibles por actividad microbiana ${ }^{(10)}$. En Colombia, se permite una adición máxima de sales de nitrito de $200 \mathrm{mg} / \mathrm{kg} /$ producto, en conjunto con inhibidores de nitrosaminas como ascorbato o eritorbato de sodio ${ }^{(11)}$. En procesados de pescado, el nitrato de sodio reacciona con aminas presentes normalmente en los peces para producir NDMA ${ }^{(12)}$. En la cerveza, también se hallan compuestos $\mathrm{N}$-nitrosos a causa del procesamiento térmico de la malta que cuenta con precursores de $\operatorname{NDMA}^{(10)}$.

De este modo, la exposición humana total a compuestos $\mathrm{N}$-nitrosos resulta de la exposición exógena o preformados endógenos. La exposición "exógena" total a los compuestos $\mathrm{N}$-nitrosos puede abarcar la exposición relacionada con los "factores del estilo de vida" (como el tabaco, los alimentos, los cosméticos, los productos para el hogar y el aire), pero también para condiciones específicas de trabajo (por ejemplo, en la industria del caucho, cuero, metal, química o afines) $)^{(10)}$. La exposición endógena, basada en la formación in vivo, depende de la captación de agentes precursores (nitrato, nitrito, óxido de nitrógeno y compuestos amino $\mathrm{N}$-nitrosables) y de la cinética de formación de compuestos $\mathrm{N}$-nitrosos en el tracto gastrointestinal ${ }^{(10)}$.

Las estimaciones internacionales de la ingesta de nitratos de los alimentos son de 31 a $185 \mathrm{mg} /$ día en Europa y en los Estados Unidos, de 40 a $100 \mathrm{mg} /$ día $^{(3)}$. La ingesta de nitritos varía de 0 a $20 \mathrm{mg} /$ día $^{(2,5)}$. Según la Autoridad Europea de Seguridad Alimentaria (EFSA), la ingesta diaria aceptable de nitrato es de $3,7 \mathrm{mg} / \mathrm{kg}$ de peso/día y para el nitrito se calculó una ingesta diaria aceptable de $0,07 \mathrm{mg} / \mathrm{kg}$ de peso/día, basada en un aumento de la metahemoglobina ${ }^{(13)}$. Es de aclarar, que las cantidades estimadas por la EFSA que pueden ser ingeridas sin presentar un riesgo apreciable para la salud, incluyen no solo la exposición a partir de vegetales, sino también, a partir de aditivos alimentarios y agua con estos compuestos.

Adicionalmente, se ha estimado que la población general consume aproximadamente $0,1 \mathrm{mg}$ de nitrosaminas por día. Dado que las nitrosaminas y sus precursores están presentes en el alimento, no se puede evitar la exposición a las nitrosaminas ${ }^{(14)}$. Aunque la principal ruta de exposición de compuestos $\mathrm{N}$-nitrosos como las nitrosaminas para la población en general es a través del consumo de alimentos y el agua, la dosis total consumida por los fumadores de cigarrillos es considerablemente mayor. Se ha estimado que los fumadores de cigarrillos pueden inhalar hasta $17 \mathrm{mg}$ de nitrosaminas por día ${ }^{(15)}$.

\section{METABOLISMO Y BIOTRANSFORMACIÓN}

Después de la ingestión oral ocurre la reducción de dos electrones para pasar de nitrato a nitrito. Esto se produce a través de la simbiosis con bacterias anaerobias facultativas que residen en las criptas de la lengua ${ }^{(16)}$. La saliva es de particular importancia ya que ahora se sabe que la bioactivación de $\mathrm{NO}_{3}$ - requiere reductasas de nitrato que residen en la cavidad bucal ${ }^{(17)}$. Doel JJ menciona que se han identificado bacterias orales Veilonella que contribuyen a la reducción oral de $\mathrm{NO}_{3}$-. Otras bacterias implicadas en la reducción de $\mathrm{NO}_{3}$ - incluyen Granulicatella adiacens, Haemophilus parainfluenzae, Actinomyces odontolyticus, Actinomyces viscosus, Actinomyces oris, Prevotella melaninogenica y Prevotella salivae ${ }^{(18)}$.

Se estima que de 5\%-7 \% de la conversión a nitritos se debe por la reducción oral de los nitratos, pudiendo ser hasta de un $20 \%$, dada la amplia presencia de bacterias reductoras de nitrato en la cavidad oral ${ }^{(19)}$. Aunque gran parte del nitrato se excreta $(60 \%-75 \%)$ en la orina directamente o después de la conversión a urea ${ }^{(20)}$, hasta el $25 \%$ es absorbido activamente por las glándulas salivales y se concentra entre 10 y 20 veces en la saliva ${ }^{(20)}$. Gran parte del nitrato sufre reabsorción tubular renal del ion, estimándose que el $96 \%$ del nitrito y nitrato filtrados se reabsorben en los túbulos renales ${ }^{(3,16)}$. Los niveles de nitrato en el plasma también aumentan considerablemente y permanecen altos durante un período prolongado (la vida media plasmática del nitrato es de 5 a 6 horas $)^{(16)}$. De manera similar ocurre con los niveles de nitrito en el plasma que aumentan luego de la ingesta de nitrato después de aproximadamente 90 minutos $^{(16)}$. 
La bioactivación de los nitritos se lleva ampliamente en el ambiente ácido gástrico, el nitrito en presencia de protones da lugar a la formación de ácido nitroso que se descompone para formar óxido nítrico $(\mathrm{ON})$ y otros óxidos de nitrógeno ${ }^{(19)}$. El nitrito no tiene que ser protonado para ser absorbido y tiene una biodisponibilidad de alrededor del $98 \%{ }^{(16,21)}$. Esta conversión de óxidos de nitrógeno que incluyen $\mathrm{ON}$ es un proceso que se ve reforzado por los polifenoles y otros agentes reductores (antioxidantes) como la vitamina C (Figura 1) ${ }^{(22)}$. El proceso comprendido desde la reducción oral del nitrato hasta la formación de óxido nítrico, representa la vía de biotransformación exógena, conocida como vía entero-salival nitrato-nitrito-óxido nítrico. La conversión gástrica de $\mathrm{ON}$ ha demostrado un rol benéfico en el mantenimiento de la integridad de la mucosa gástrica al favorecer el aumento del flujo sanguíneo y la secreción de moco. En cambio, se ha expuesto que los medicamentos inhibidores de la bomba de protones, que apuntan a la reducción del ácido gástrico, pueden estar relacionados con la enfermedad cardiovascular, en parte, por la alteración en la conversión y bioactivación de nitrato-nitrito-ON ${ }^{(19)}$.
Igualmente, el $\mathrm{ON}$ es generado de manera endógena mediante la vía de la L-arginina-NOS a partir de óxido nítrico sintasas (NOS) y sustratos como L-arginina y oxígeno molecular. Asimismo, para esta conversión se hace necesaria la presencia de cofactores como la nicotinamida adenina dinucleótido fosfato en su forma reducida $(\mathrm{NADPH})$ y la tetrahidrobiopterina $(\mathrm{BH} 4)$. La oxidación y alteración de la función de NOS, así como una baja disponibilidad de cofactores y sustratos al parecer son factores implicados en el desarrollo de la enfermedad cardiovascular ${ }^{(19)}$.

Si el estómago presenta ciertos tipos de aminas como las secundarias u otro tipo de aminoácidos, puede reaccionar para generar nitrosaminas (Figura 1) ${ }^{(22)}$. Esta reacción se favorece en condiciones ácidas (como en el estómago) y con calor (como cuando se cocina a altas temperaturas alimentos proteicos, como durante la fritura $\mathrm{u}$ horneado $)^{(5)}$. En todos los casos, se absorben bien en el tracto gastrointestinal. Otras rutas de absorción abarcan la inhalación o exposición dérmica, que conduce a tasas de captación similares a la de la vía oral. Las $\mathrm{N}$-nitrosaminas se distribuyen con el torrente sanguíneo y se metaboliza rápidamente, predominantemente en el hígado ${ }^{(10)}$.

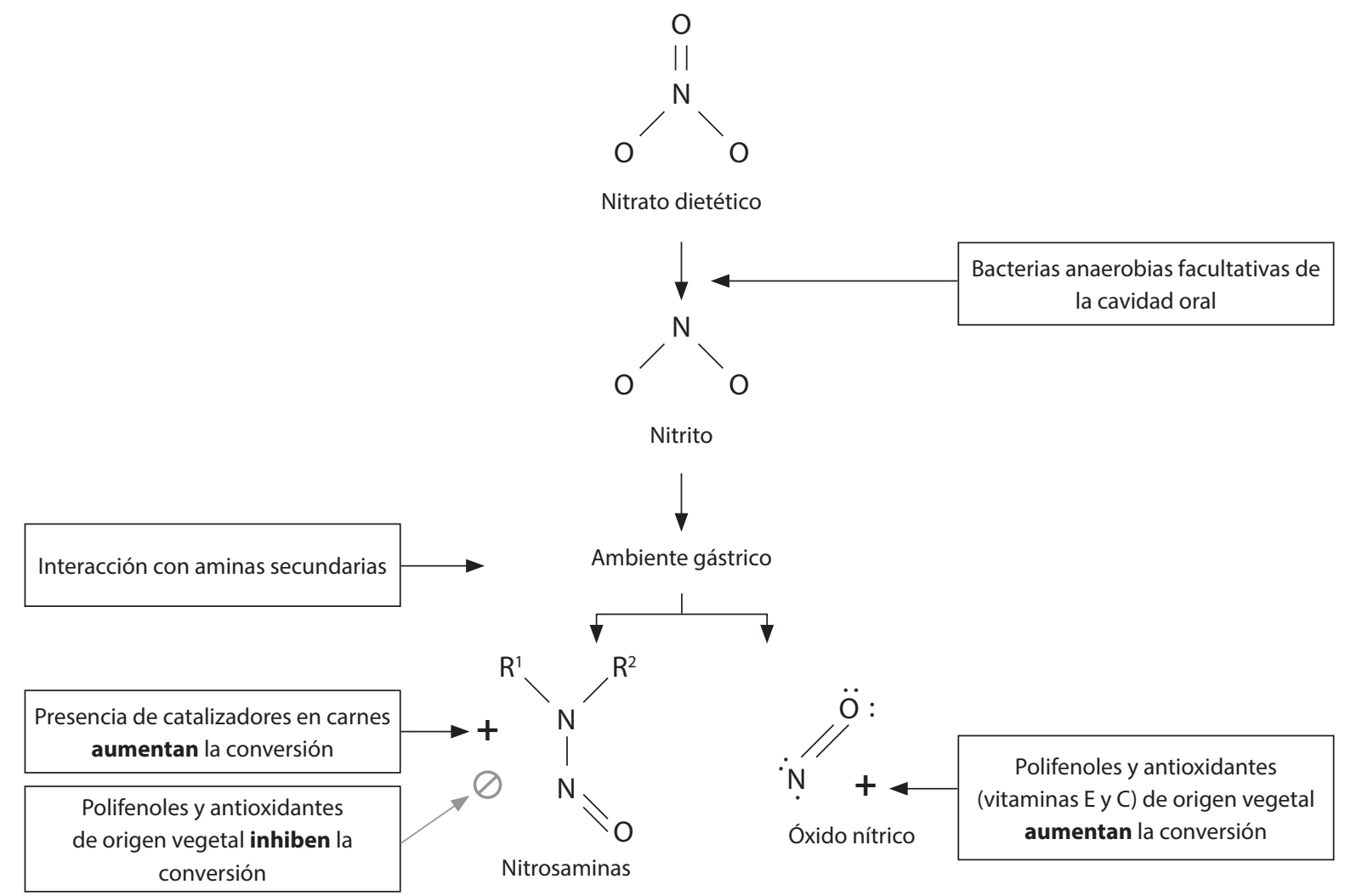

Figura 1. Metabolismo y biotransformación de nitratos/nitritos: vía entero-salival nitrato-nitrito-ON. llustración realizada por el autor. 
Las N-nitrosaminas requieren de activación metabólica, que consiste en la hidroxilación en la posición carbono alfa $(\alpha-C)$, mediada por el citocromo P450 dependiente de monooxigenasas. Esto da como resultado la formación de una $\alpha$-hidroxi-N-nitrosamina, que es inestable y que libera un aldehído para formar una monoalquilnitrosamina aún más inestable ${ }^{(10)}$. Este último se reorganiza en el correspondiente diazonio intermedio que reacciona como un electrófilo con biomoléculas como el ácido desoxirribonucleico $(\mathrm{ADN})$, ácido ribonucleico (ARN), proteínas o glutatión, formando aductos covalentes con centros nucleófilos apropiados. Debido a la expresión variable de las isoenzimas del citocromo P450 en diferentes órganos, los compuestos N-nitrosos también pueden sufrir biotransformación en tejidos extrahepáticos como pulmón, tracto gastrointestinal $\mathrm{u}$ otros órganos ${ }^{(10)}$.

Las $\mathrm{N}$-nitrosaminas con cadenas laterales más largas también pueden ser hidroxiladas en posiciones distintas al átomo de $\alpha$-C. Los metabolitos hidroxilados resultantes pueden ser excretados directamente u oxidados adicionalmente a los respectivos ácidos o compuestos carbonílicos, o pueden experimentar reacciones de conjugación por enzimas de fase II, los conjugados resultantes se excretan por la vía biliar o la orina ${ }^{(10)}$. Los compuestos electrofílicos reactivos generados metabólicamente conducen a la alquilación de bases de $\mathrm{ADN}$, específicamente la alquilación del alquilo guanina $\mathrm{O} 6$; el alquilo timidina $\mathrm{O} 4$ y el $\mathrm{O} 2$ dan lugar a errores de emparejamiento del ADN y errores de codificación que representan lesiones mutagénicas ${ }^{(10)}$.

\section{IMPACTO EN LA SALUD}

\section{Cáncer}

Según Habermeyer, el conocimiento sobre los compuestos nitrosos se ha acumulado en estudios desde principios de los años 50 hasta la actualidad ${ }^{(10)}$. Barnes y Magee describieron el fuerte potencial carcinogénico del NDMA en $1956^{(10)}$. Habermeyer mencionó que en los años 90 se encontró que la carcinogenicidad y otros efectos biológicos dependían de la naturaleza del grupo sustituyente de cadenas laterales de aminoácidos (R1 y R2) ${ }^{(10,14)}$.

El cáncer es el riesgo de salud más importante que se ha asociado históricamente con el nitrato y el nitrito. Aunque estos compuestos no son carcinógenos en sí mismos, tienen el potencial de reaccionar con otros compuestos dentro de los alimentos durante la cocción o en el tracto digestivo para formar compuestos $\mathrm{N}$-nitrosos como las nitrosaminas ${ }^{(5)}$. La exposición crónica y continua a dosis bajas de nitrosaminas en la dieta se considera de importancia toxicológica para los seres humanos, porque pueden producir cáncer en diversos órganos y tejidos, incluidos los pulmones, el cerebro, el hígado, el riñón, la vejiga, el estómago, el esófago y el seno nasal ${ }^{(14)}$. El $75 \%-80 \%$ de las nitrosaminas analizadas son carcinógenas para los mamíferos ${ }^{(14)}$. La dimetilnitrosamina, un miembro de la familia de las nitrosaminas presente en los alimentos, es altamente carcinogénica para el hígado y los riñones en casi todas las especies de mamíferos analizadas ${ }^{(14)}$. Se consideran que las nitrosaminas derivadas del tabaco son uno de los principales agentes causantes de cáncer que se encuentran en el humo del tabaco y en sus productos ${ }^{(14)}$.

Las nitrosaminas requieren bioactivación para ser carcinogénicas. Se sabe que la activación procede primero por la hidroxilación de un carbono y se forma una nitrosamina primaria inestable, que finalmente se tautomeriza a un ion carbonio ${ }^{(14)}$. Este ion altamente reactivo se alquila fácilmente con macromoléculas celulares cercanas. El cáncer y la mutagenicidad se desarrollan cuando los metabolitos reactivos de la nitrosamina reaccionan con macromoléculas genéticas ${ }^{(14)}$.

\section{Metahemoglobinemia}

Otro factor de riesgo asociado con la ingesta de nitratos y nitritos es la metahemoglobinemia. Esta condición puede ocurrir cuando el nitrito reacciona con la hemoglobina, lo que hace que sea incapaz de transportar oxígeno. La anemia bioquímica resultante conduce a una cianosis característica $^{(23)}$. El riesgo de metahemoglobinemia se da principalmente en niños menores de 3 meses de edad, por una menor actividad de la enzima dinucleótido de nicotinamida y adenina (NADH)-citocromo B5 reductasa; no obstante, para este grupo de edad no se recomienda la ingesta de vegetales ni otro tipo de alimentos, pues la prioridad es preservar la lactancia materna exclu$\mathrm{siva}^{(23)}$. En cuanto a los adultos, se han reportado algunos casos de metahemoglobinemia por la ingesta de grandes cantidades de vegetales que han pasado por procesos inadecuados de almacenamiento y conservación; sin embargo, se considera que es segura la ingesta de vegetales con altos contenidos de nitrato, siempre y cuando se hayan preparado y almacenado correctamente ${ }^{(23)}$.

\section{TRASTORNOS DE LA GLÁNDULA TIROIDEA}

Se ha descrito en la literatura un efecto "anti-tiroideo" asociado a la ingesta de nitrato/nitrito, especialmente en regiones que se caracterizan por presentar altas con- 
centraciones de nitrato en el agua. En este sentido, se ha sugerido que la alta exposición a estos compuestos puede alterar la captación de yodo y favorecer trastornos de la glándula tiroidea. Una revisión sistemática y metaanálisis ${ }^{(24)}$ recopiló diferentes estudios en animales y en humanos con el objetivo de determinar si la exposición a nitrato/nitrito es un factor de riesgo para la disfunción tiroidea. Las investigaciones en animales indican que el nitrato inorgánico puede actuar como un agente inhibidor de la captación de yodo y que la exposición crónica puede conducir, entre otras cosas, a bocio e hipertrofia de la glándula tiroidea. Sin embargo, las dosis empleadas en la mayor parte de estos estudios, excedían entre 10 y 600 veces la ingesta diaria aceptable estipulada por la EFSA, administrando incluso, dosis de hasta de 1500 y $3000 \mathrm{mg} / \mathrm{kg} / \mathrm{d}$, inhabilitando, por tanto, la extrapolación de estos resultados en humanos $^{(24)}$. En relación a los ensayos clínicos recopilados, se emplearon dosis de dos a cuatro veces superiores a la ingesta diaria aceptable en humanos, no encontrando efecto alguno sobre la captación de yodo, ni en las concentraciones plasmáticas de triyodotironina (T3), tiroxina (T4) y tirotropina (TSH) ${ }^{(24)}$.

$\mathrm{Si}$ bien algunos estudios epidemiológicos han reportado efectos deletéreos sobre la glándula tiroidea, asociados a la ingesta de agua contaminada con nitratos, aún se encuentran inconsistencias para establecer una asociación significativa. Además, se sugiere que el efecto del nitrato sobre la función tiroidea puede estar determinado por la ingesta de yodo y que solo en sujetos con deficiencia nutricional de yodo podría ser considerable ${ }^{(24)}$.

\section{ÓXIDO NÍTRICO (ON)}

Los efectos deletéreos atribuidos a la ingesta de nitratos, específicamente su relación con el riesgo de desarrollar cáncer, parecen ser dependientes de la procedencia de los mismos ${ }^{(19)}$. En este sentido, se ha descrito que la ingesta de nitratos en presencia de compuestos bioactivos y antioxidantes como los polifenoles y las vitaminas $\mathrm{E}$ y $\mathrm{C}$, abundantes en alimentos de origen vegetal, inhiben su capacidad de conversión a $\mathrm{N}$-nitrosaminas. Además, sorprendentemente, algunos autores sugieren que la ingesta de nitratos a partir de alimentos de origen vegetal no aumenta el riesgo de mortalidad por cáncer $y$, por el contrario, se relaciona con efectos protectores frente al mismo ${ }^{(19)}$. Un claro ejemplo de este planteamiento es la reconocida dieta mediterránea, un patrón alimentario comprendido en gran medida por la ingesta de vegetales y otros alimentos ricos en nitrato ${ }^{(25)}$ y cuya adherencia se asocia de manera importante con un menor riesgo de padecer cualquier tipo de cáncer ${ }^{(26)}$.

Los nitratos procedentes de fuentes naturales como los vegetales no solo no se encuentran asociados con los riesgos que históricamente se les ha atribuido, sino que también hay un interés en la posibilidad de que el nitrato inorgánico (dietético $\left[\mathrm{NO}_{3}^{-}\right]$) pueda ser uno de los componentes que proporcione efectos protectores de una dieta alta en frutas y verduras ${ }^{(27)}$. Se cree que este beneficio propuesto se deriva de su capacidad para elevar los niveles de ON. Esta posibilidad ha sido propuesta ya que los vegetales de hojas verdes son particularmente altos en el anión $\mathrm{NO}_{3}$ - que puede terminar como $\mathrm{ON}^{(28)}$. Además, está la hipótesis de que los antioxidantes presentes en los vegetales pueden hacer que esos productos tengan menos probabilidades de causar efectos dañinos y más probabilidades de causar efectos saludables ${ }^{(2)}$.

El ON se produce en las células endoteliales que recubren todos los vasos sanguíneos y los vasos linfáticos de todo el cuerpo, también dentro del sistema nervioso y el sistema inmunológico ${ }^{(16)}$. $\mathrm{El} \mathrm{ON}$ es un potente vasodilatador, un inhibidor de la agregación plaquetaria y la especie activa de la nitroglicerina. El ON es sintetizado por células de mamíferos a partir de L-arginina a través de una reacción de oxidación compleja catalizada por las enzimas NOS. Se ha establecido que la producción endógena de ON desempeña un papel importante en la homeostasis vascular, la neurotransmisión y los mecanismos de defensa del huésped. De hecho, el ON es una de las moléculas más importantes producidas dentro del cuerpo humano ${ }^{(16)}$.

\section{SALUD CARDIOVASCULAR}

Los beneficios potenciales del nitrato y $\mathrm{ON}$ en el sistema cardiovascular y en condiciones isquémicas se han relacionado por sus efectos antiinflamatorios ${ }^{(29)}$, antihipertensivos ${ }^{(30)}$, antiplaquetarios y antihipertrófi$\cos ^{(31)}$. Adicionalmente, se han observado efectos benéficos sobre la presión arterial ${ }^{(32)}$. El consumo de 0,1 $\mathrm{mmol} / \mathrm{kg}$ de peso corporal de nitrato (595 mg para un adulto de $70 \mathrm{~kg}$ ) durante 3 días puede reducir significativamente la presión arterial diastólica (PAD), que fue en promedio 3,7 $\mathrm{mm} \mathrm{Hg}$ más baja después de la suplementación con nitrato que después de la suplementación con placebo, y la presión arterial media (PAM) fue 3,2 mm Hg más baja ${ }^{(32)}$.

En un metaanálisis se ha demostrado que el aumento de la ingesta dietética de vegetales ricos en nitratos es eficaz en la reducción de la presión arterial ${ }^{(32)}$. Para 
obtener niveles de nitrato suficientes para el manejo de la presión arterial, se debe proporcionar un mínimo de 450 a $550 \mathrm{mg}$ de nitrato al menos 90 minutos antes para permitir tiempo suficiente para la circulación enterosalival y la reducción a nitrito por las bacterias orales. Se ha demostrado que el nitrato disminuye la presión arterial a partir de los 90 minutos y dura varias horas ${ }^{(33)}$.

Los efectos cardioprotectores del ON como la inducción de la proliferación de células musculares lisas vasculares, angiogénesis, disminución de la presión arterial sistólica (PAS) y PAD, mejora de la función endotelial, aumento del flujo sanguíneo, disminución de la rigidez arterial y disminución de la agregación plaquetaria han llevado a que actualmente se conciban los nitratos de origen vegetal como compuestos bioactivos o fitoquímicos ${ }^{(34,35)}$. En este sentido, y como se mencionó previamente, se ha demostrado que algunas de las dietas que exhiben efectos protectores frente a enfermedades crónicas, como la dieta mediterránea, son ricas en nitrato inorgánico; por el contrario, una baja ingesta de nitratos puede limitar la biodisponibilidad de ON, lo cual se asocia con alteración de la función endotelial, relacionada a su vez con un mayor riesgo de enfermedad cardiovascular e incluso enfermedad renal ${ }^{(19)}$.

\section{RENDIMIENTO DEPORTIVO}

Para la mayoría de los estudios en humanos, el zumo de remolacha ha sido la fuente de nitrato de elección. Sin embargo, para obtener niveles de nitrato suficientes para mejorar el rendimiento ${ }^{(36)}$, se debe proporcionar un mínimo de $500 \mathrm{~mL}$ de jugo de remolacha al menos 2,5 horas antes del ejercicio para permitir suficiente tiempo para la circulación entero-salival y la reducción a nitrito ${ }^{(25)}$. Las investigaciones indican que elevar los niveles de nitrato y nitrito en el cuerpo antes del ejercicio mediante el consumo de nitrato en la dieta puede aumentar la producción de ON, la eficiencia del oxígeno y el rendimiento del ejercicio. El consumo de $500 \mathrm{~mL}$ de jugo de remolacha, que es alto en nitrato dietético (527 mg), 2,5 horas antes del ejercicio mejoró la potencia y el rendimiento de 9 ciclistas masculinos competitivos de nivel club durante una prueba de ciclismo de $4 \mathrm{~km}$ y $16,1 \mathrm{~km}$ comparado con el placebo. Además, la suplementación dietética con jugo de remolacha, que contiene aproximadamente $400 \mathrm{mg}$ de nitrato por dosis, dos veces al día ( $816 \mathrm{mg}$ en total), aumentó la concentración de nitrito en el plasma, redujo la presión arterial e influyó positivamente en las respuestas fisiológicas al ejercicio ${ }^{(3)}$.

\section{OTROS ROLES POTENCIALES}

Más allá de los efectos benéficos que ejercen los nitratos y el $\mathrm{ON}$ en la salud cardiovascular y en el rendimiento deportivo, diferentes investigaciones han sugerido que estos compuestos presentan un rol clave en el adecuado funcionamiento del sistema nervioso ${ }^{(37)} \mathrm{y}$ en la prevención del cáncer ${ }^{(38)}$ : al parecer, el ON favorece la plasticidad sináptica, la vasodilatación cerebral, potencia la neurotransmisión, regula el comportamiento, el ciclo del sueño, la defensa del sistema nervioso central, inhibe la apoptosis neuronal y protege frente al estrés oxidativo, con un subsecuente impacto positivo en la memoria y la cognición $^{(39)}$. En cuanto al cáncer, altas concentraciones de ON exhiben efectos citotóxicos y proapoptóticos, lo que favorece acciones tumoricidas. El efecto contrario se halla en presencia de bajas concentraciones ${ }^{(39)}$.

\section{CONCLUSIÓN}

A pesar del vínculo que se ha establecido por años entre la ingesta de nitrato/nitrito y un mayor riesgo de padecer cáncer, por la formación de nitrosaminas resultante de la interacción de estos compuestos, con altas temperaturas o con el ambiente gástrico, no debe desconocerse la capacidad que tienen estos compuestos de derivar en ON. Este último es esencial para el mantenimiento de la salud cardiovascular y metabólica, además de los beneficios que se le atribuyen en relación con la salud cognitiva, el rendimiento deportivo y potenciales acciones tumoricidas. Los efectos en la salud derivados de la ingesta de nitrato/nitrito dependerán de manera importante de factores como su procedencia. En este sentido, la ingesta de nitratos que se encuentran naturalmente en alimentos de origen vegetal, no supone un riesgo para la salud y, por el contrario, muchos de los efectos benéficos que se le atribuyen a la ingesta de vegetales, como un menor riesgo de padecer enfermedades crónicas, tienen que ver con su aporte de estos compuestos. Por su parte, los patrones alimentarios más reconocidos con beneficios en torno a la salud humana, como la dieta mediterránea, son particularmente altos en nitratos. Estos hallazgos se deben a que la presencia de nitrato/nitrito en alimentos de origen vegetal se acompaña adicionalmente de inhibidores de la formación de compuestos $\mathrm{N}$-nitrosos y potencializadores de la formación de $\mathrm{ON}$, como los polifenoles $\mathrm{y}$ algunas vitaminas antioxidantes. Por tanto, la presencia de inhibidores o catalizadores de las reacciones de nitrosación en los alimentos se consolida como un 
segundo factor importante al momento de determinar el riesgo que los nitratos y nitritos puedan representar. En algunos productos, como los cárnicos procesados, se hallan compuestos nitrogenados que pueden actuar como agentes nitrosantes e inducir la formación de compuestos nitrosos.

En conclusión, a la luz de la evidencia disponible, se puede afirmar que el aporte de nitrato/nitrito en alimentos vegetales no representa un riesgo para la salud en relación con el cáncer u otras enfermedades crónicas; por el contrario, podrían prevenir su aparición. Parece ser recomendable mantener mesura en la ingesta de nitrato/nitrito a partir de procesados como los productos cárnicos.

\section{Financiación}

Los autores no recibieron financiación específica para la redacción de este artículo.

\section{Conflicto de intereses}

Los autores no manifiestan ningún conflicto de interés que declarar.

\section{Declaración de autoría}

MLP, BGR participaron en la redacción del artículo. Todos los autores revisaron el artículo y validaron su versión final.

\section{Referencias bibliográficas}

1. Kalaycıoğlu Z, Erim FB. Nitrate and Nitrites in Foods: Worldwide Regional Distribution in View of Their Risks and Benefits. J Agric Food Chem. 2019;67(26):7205-22. doi: 10.1021/acs.jafc.9b01194.

2. Hord NG, Tang Y, Bryan NS. Food sources of nitrates and nitrites: the physiologic context for potential health benefits. Am J Clin Nutr. 2009;90(1):1-10. doi: 10.3945/ajcn.2008.27131.

3. Bryan NS. Nitrites and Nitrates. En: Caballero B, Finglas P, Toldra F (editores). Encyclopedia of Food and Health. Elsevier; 2016. p. 73-78. doi: 10.1016/B978-0-12-3849472.00484-0.

4. Iammarino M, Di Taranto A, Cristino M. Monitoring of nitrites and nitrates levels in leafy vegetables (spinach and lettuce): a contribution to risk assessment. J Sci Food Agric. 2014;94(4):773-8. doi: 10.1002/jsfa.6439.

5. Bedale W, Sindelar JJ, Milkowski AL. Dietary nitrate and nitrite: Benefits, risks, and evolving perceptions. Meat Sci. 2016;120:85-92. doi: 10.1016/j.meatsci.2016.03.009.
6. Raczuk J, Wadas W, Głozak K. Nitrates and nitrites in selected vegetables purchased at supermarkets in Siedlce, Poland. Rocz Panstw Zakl Hig. 2014;65(1):15-20.

7. Griesenbeck JS, Steck MD, Huber JC, Sharkey JR, Rene AA, Brender JD. Development of estimates of dietary nitrates, nitrites, and nitrosamines for use with the short willet food frequency questionnaire. Nutr J. 2009;8:16. doi: 10.1186/14752891-8-16.

8. Karwowska M, Kononiuk A. Nitrates/Nitrites in FoodRisk for Nitrosative Stress and Benefits. Antioxidants. 2020;9(3):241. doi: 10.3390/antiox9030241.

9. Ward MH, Jones RR, Brender JD, de Kok TM, Weyer PJ, Nolan BT, et al. Drinking Water Nitrate and Human Health: An Updated Review. Int J Environ Res Public Health. 2018;15(7):1557. doi: 10.3390/ijerph15071557.

10. Habermeyer M, Eisenbrand G. N-Nitroso Compounds in Foods. En: Varelis P, Melton L, Shahidi F. Encyclopedia of Food Chemistry. Elsevier; 2019. p. 593-602. doi: 10.1016/ B978-0-08-100596-5.21824-6.

11. NTC 1325: Productos cárnicos procesados no enlatados [Internet]. ICONTEC; 2008 [Fecha de consulta: 10 de junio de 2020]. Disponible en: https://tienda.icontec.org/gpindustrias-alimentarias-productos-carnicos-procesados-noenlatados-ntc1325-2008.html.

12. Zeilmaker MJ, Bakker MI, Schothorst R, Slob W. Risk Assessment of $\mathrm{N}$-nitrosodimethylamine Formed Endogenously after Fish-with-Vegetable Meals. Toxicol Sci. 2010;116(1):323-35. doi: 10.1093/toxsci/kfq093.

13. Nitrites and nitrates added to food [Internet]. EFSA; 2017 [Fecha de consulta: 10 de mayo de 2020]. Disponible en: https://www.efsa.europa.eu/sites/default/files/corporate_ publications/files/nitrates-nitrites-170614.pdf.

14. Robles H. Nitrosamines. En: Wexler P. Encyclopedia of Toxicology. Elsevier; 2014. p. 584-5. doi: 10.1016/B978-012-386454-3.00523-6.

15. Konstantinou E, Fotopoulou F, Drosos A, Dimakopoulou N, Zagoriti Z, Niarchos A, et al. Tobacco-specific nitrosamines: A literature review. Food Chem Toxicol. 2018;118:198-203. doi: 10.1016/j.fct.2018.05.008.

16. Bryan NS. An Overview of Nitrite and Nitrate. En: Bagchi D. Sustained Energy for Enhanced Human Functions and Activity. Elsevier; 2017. p. 53-65. doi: 10.1016/B978-0-12805413-0.00003-X.

17. Lundberg JO, Weitzberg E, Cole JA, Benjamin N. Nitrate, bacteria and human health. Nat Rev Microbiol. 2004;2(7):593602. doi: $10.1038 /$ nrmicro929.

18. Doel JJ, Benjamin N, Hector MP, Rogers M, Allaker RP. Evaluation of bacterial nitrate reduction in the human oral cavity. Eur J Oral Sci. 2005;113(1):14-9. doi: 10.1111/j.16000722.2004.00184.x.

19. Bondonno CP, Blekkenhorst LC, Liu AH, Bondonno NP, Ward NC, Croft KD, et al. Vegetable-derived bioactive nitrate 
and cardiovascular health. Mol Aspects Med. 2018;61:83-91. doi: 10.1016/j.mam.2017.08.001.

20. Pannala AS, Mani AR, Spencer JPE, Skinner V, Bruckdorfer $\mathrm{KR}$, Moore KP, et al. The effect of dietary nitrate on salivary, plasma, and urinary nitrate metabolism in humans. Free Radic Biol Med. 2003;34(5):576-84. doi: 10.1016/s08915849(02)01353-9.

21. Hunault CC, van Velzen AG, Sips AJAM, Schothorst RC, Meulenbelt J. Bioavailability of sodium nitrite from an aqueous solution in healthy adults. Toxicol Lett. 2009;190(1):48-53. doi: 10.1016/j.toxlet.2009.06.865.

22. Bryan NS, Alexander DD, Coughlin JR, Milkowski AL, Boffetta P. Ingested nitrate and nitrite and stomach cancer risk: An updated review. Food Chem Toxicol. 2012;50(10):364665. doi: 10.1016/j.fct.2012.07.062.

23. Chan TYK. Vegetable-borne nitrate and nitrite and the risk of methaemoglobinaemia. Toxicol Lett. 2011;200(1-2):107-8. doi: 10.1016/j.toxlet.2010.11.002.

24. Bahadoran Z, Mirmiran P, Ghasemi A, Kabir A, Azizi F, Hadaegh F. Is dietary nitrate/nitrite exposure a risk factor for development of thyroid abnormality? A systematic review and meta-analysis. Nitric Oxide. 2015;47:65-76. doi: 10.1016/j. niox.2015.04.002.

25. Shannon OM, Stephan BCM, Minihane A-M, Mathers JC, Siervo M. Nitric Oxide Boosting Effects of the Mediterranean Diet: A Potential Mechanism of Action. J Gerontol A Biol Sci Med Sci. 2018;73(7):902-4. doi: 10.1093/gerona/gly087.

26. Schwingshackl L, Schwedhelm C, Galbete C, Hoffmann G. Adherence to Mediterranean Diet and Risk of Cancer: An Updated Systematic Review and Meta-Analysis. Nutrients. 2017;9(10):1063. doi: 10.3390/nu9101063.

27. Kapil V, Webb AJ, Ahluwalia A. Inorganic nitrate and the cardiovascular system. Heart. 2010;96(21):1703-9. doi: 10.1136/hrt.2009.180372.

28. Rathod KS, Velmurugan S, Ahluwalia A. A 'green' diet-based approach to cardiovascular health? Is inorganic nitrate the answer? Mol Nutr Food Re. 2016;60(1):185-202. doi: $10.1002 / \mathrm{mnfr} .201500313$.

29. Ashworth A, Mitchell K, Blackwell JR, Vanhatalo A, Jones AM. High-nitrate vegetable diet increases plasma nitrate and nitrite concentrations and reduces blood pressure in healthy women. Public Health Nutr. 2015;18(14):2669-78. doi: 10.1017/S1368980015000038.

30. Sobko T, Marcus C, Govoni M, Kamiya S. Dietary nitrate in Japanese traditional foods lowers diastolic blood pressure in healthy volunteers. Nitric Oxide. 2010;22(2):136-40. doi: 10.1016/j.niox.2009.10.007.

31. Hobbs DA, George TW, Lovegrove JA. The effects of dietary nitrate on blood pressure and endothelial function: a review of human intervention studies. Nutr Res Rev. 2013;26(2):21022. doi: 10.1017/S0954422413000188.

32. Siervo M, Lara J, Ogbonmwan I, Mathers JC. Inorganic Nitrate and Beetroot Juice Supplementation Reduces Blood Pressure in Adults: A Systematic Review and Meta-Analysis. J Nutr. 2013;143(6):818-26. doi: 10.3945/jn.112.170233.

33. Coles LT, Clifton PM. Effect of beetroot juice on lowering blood pressure in free-living, disease-free adults: a randomized, placebo-controlled trial. Nutr J. 2012;11:106. doi: 10.1186/1475-2891-11-106.

34. Farah C, Michel LYM, Balligand J-L. Nitric oxide signalling in cardiovascular health and disease. Nat Rev Cardiol. 2018;15(5):292-316. doi: 10.1038/nrcardio.2017.224.

35. Tarkin JM, Kaski JC. Vasodilator Therapy: Nitrates and Nicorandil. Cardiovasc Drugs Ther. 2016;30(4):367-78. doi: 10.1007/s10557-016-6668-z.

36. Menezes EF, Peixoto LG, Teixeira RR, Justino AB, Puga GM, Espindola FS. Potential Benefits of Nitrate Supplementation on Antioxidant Defense System and Blood Pressure Responses after Exercise Performance. Oxid Med Cell Longev. 2019;2019:7218936. doi: 10.1155/2019/7218936.

37. Cossenza M, Socodato R, Portugal CC, Domith ICL, Gladulich LFH, Encarnação TG, et al. Nitric Oxide in the Nervous System: Biochemical, Developmental, and Neurobiological Aspects. Vitam Horm. 2014;96:79-125. doi: 10.1016/B978-0-12-800254-4.00005-2.

38. Huang Z, Fu J, Zhang Y. Nitric Oxide Donor-Based Cancer Therapy: Advances and Prospects. J Med Chem. 2017;60(18):7617-35. doi: 10.1021/acs.jmedchem.6b01672.

39. Maher A, Rahman MFA, Gad MZ. The Role of Nitric Oxide from Neurological Disease to Cancer. Adv Exp Med Biol. 2017;1007:71-88. doi: 10.1007/978-3-319-60733-7_5. 